\title{
Better Management of Adverse Events Favors Sorafenib Treatment of HCC Patients and Impact on Survival
}

\author{
Regiane S. S. M. Alencar ${ }^{1}$, Luciana Kikuchi ${ }^{1}$, Cláudia M. Tani ${ }^{1}$, Aline L. Chagas ${ }^{1}$, \\ Cinira C. Camargo"1, Túlio E. F. Pfiffer ${ }^{2}$, Paulo M. G. Hoff ${ }^{2}$, Flair J. Carrilho ${ }^{1}$ \\ ${ }^{1}$ São Paulo Clínicas Liver Cancer Group, Instituto do Câncer do Estado de São Paulo (ICESP), Hospital das \\ Clínicas, Department of Gastroenterology, University of São Paulo School of Medicine, São Paulo, Brazil \\ ${ }^{2}$ Medical Oncology, Instituto do Câncer do Estado de São Paulo (ICESP), São Paulo, Brazil \\ Email: ${ }^{2}$ rssmalencar@gmail.com
}

Received 4 March 2016; accepted 19 April 2016; published 22 April 2016

Copyright (C) 2016 by authors and Scientific Research Publishing Inc.

This work is licensed under the Creative Commons Attribution International License (CC BY). http://creativecommons.org/licenses/by/4.0/

\section{Abstract}

Introduction: Sorafenib is an orally active multikinase inhibitor approved for the treatment of advanced hepatocellular carcinoma (HCC) and is the only systemic treatment associated with a survival benefit in advanced stage. The aims of this study were to evaluate the tolerance and survival of sorafenib-treated patients, and to identify potential prognostic factors of survival. Methods: A total of $88 \mathrm{HCC}$ patients treated with sorafenib from June 2010 to January 2014 were retrospectively reviewed. Tumour stage, liver function and adverse events to sorafenib were analyzed. Univariate and multivariate analysis were carried out to identify predictors of survival in patients with advanced HCC treated with sorafenib. Results: There were $64(73 \%)$ males included in this study, with a median age of 61.16 years. Eight (91\%) patients had Child-Pugh class A cirrhosis. Most patients were classified as BCLC C $(82 \%)$. Hepatitis C virus was the predominant cause of HCC $(68 \%)$. Sorafenib was the initial treatment modality in $43 \%$. Median time of sorafenib therapy was 8.23 months. Overall survival in 1 year was $57.3 \%$ and $36.7 \%$ in 2 years. The median survival was 16.3 months. In the univariate analysis of the OS based on Child-Pugh score did not demonstrate a significant difference in our study $(p=0.62)$. The presence of dermatologic adverse event predicted a better overall survival in the multivariate analysis. Better survival was also observed in patients with AFP level $<100 \mathrm{ng} / \mathrm{ml}$ in the start of sorafenib therapy $(p=0.001)$. Patients that used Sorafenib for $\geq 6$ months had shown better outcome. None of the patients discontinued sorafenib because of adverse effects. Conclusions: Advanced HCC patients treated with sorafenib who experienced dermatologic adverse event and low AFP level $<100 \mathrm{ng} / \mathrm{ml}$ showed better overall survival. As expected, longer sorafenib therapy ( $\geq 6$ months) was associated to better survival. Even in the presence of adverse events, the use of sorafenib should be continued because the longer "Corresponding author. 
usage time improves survival.

Keywords

Management of Adverse Event, Hepatocellular Carcinoma, Sorafenib Therapy, Predictors of
Overall Survival, Dermatologic Adverse Event

\section{Introduction}

Hepatocellular carcinoma (HCC) is one of the most common malignancies and leading causes of cancer-related death globally [1]. In 2008, approximately 750,000 patients were diagnosed with HCC and more than 695,000 patients died from the disease. Although patients with early-stage HCC may potentially be cured by surgery, local ablation, or liver transplantation, many patients eventually develop or present with metastatic or locally advanced diseases that are not amenable to curative locoregional therapies. For decades, prognosis of such patients has been reported as being extremely poor, yielding a median survival time in the range of 4 - 7 months [2]-[4].

Sorafenib improves the overall survival (OS) of patients with advanced hepatocellular carcinoma (HCC) with a good safety profile and it is the first molecular target treatment approved for HCC therapy [3]. It decreases the risk of death by 31\% (Hazard Ratio 0.69) and its impact in the OS of patients with HCC is maintained regardless of race, etiology, and the baseline characteristics of patients [5] [6].

The SHARP trial demonstrated a prolonged median overall survival from 7.9 to 10.7 months and time to progression from 2.8 to 5.5 months compared with placebo in unresectable advanced HCC [3]. The efficacy of sorafenib in the Eastern world was also confirmed through the Asia-Pacific study [4]. However, a substantial portion of sorafenib-treated patients showed no response to sorafenib, with a disease-control rate of 43\% [3]. Adverse effects of the drugs, may decrease the compliance to the best treatment available [6]. Therefore, determining patients that are mostly likely to benefit from sorafenib is very important. Investigators have worked to find the biomarkers that predict patient survival or response to sorafenib $(7-10)$. Through analysis of serological markers in patients who participated in the SHARP trial, serum concentrations of VEGF and angiopoietin-2 proved to be a predictor of patient survival, but not of response to treatment [7]. Zhang et al. reported that phosphorylated ERK is a potential predictive biomarker of sensitivity to sorafenib when treating advanced HCC patients [8]. Pinter et al. reported that baseline AST level may be a potential predictive factor in determining the optimal patient for treatment with sorafenib in Child-Pugh B cirrhosis patients with advanced HCC [9]. Increased OS in patients, with skin toxicity was reported by Otsuka et al. [10]. Excluding the SHARP trial, the results of the studies have limited number of patients with inconsistent results.

Despite the analysis of different biomarkers [7] and/or functional radiologic evaluation in this population, it has been unfeasible to identify those patients that benefit most from this treatment. Patients with prolonged survival after therapy for advanced HCC are relatively uncommon and a long-term disease-free status remains rare. Several case reports have indicated that effective systemic therapy using sorafenib or anti-angiogenic therapies combined with aggressive locoregional treatment may prolong survival in selected patients [11]-[13]. However, the characteristics of patients with prolonged survival after therapy for advanced HCC have not been systematically evaluated. The aims of this study were to evaluate the tolerance and survival of sorafenib-treated patients, and to identify potential prognostic factors of survival.

\section{Patients and Methods}

\subsection{Patients}

A total of 88 HCC patients treated with sorafenib from June 2010 to January 2014 were followed in outpatient clinics of Division of Gastroenterology and Hepatology, Department of Gastroenterology, University of São Paulo School of Medicine, Hospital das Clínicas, Instituto do Cancer do Estado de São Paulo, independent of the stage of BCLC. The majority of patients was Child-Pugh A disease and were asymptomatic. In the beginning of sorafenib therapy, 72 patients were classified as BCLC C and 16 patients were BCLC B who failed or presented contraindication to locoregional treatment or presented progressive disease. 
HCC was diagnosed according to the European Association criteria for Liver Studies (EASL) and the American Association for Study of the Liver (AASLD) [14] [15].

Clinical data and radiological findings were reviewed retrospectively, conducted by a single researcher. Demographic data, liver disease and tumor characteristics, duration of therapy, AEs, and survival information were collected. As well as ECOG-PS (Eastern Cooperative Oncology Group-Performance Status) [16] and Liver function according to the score of Child-Pugh [17].

\subsection{Sorafenib Treatment}

All the patients received $400 \mathrm{mg}$ of sorafenib twice daily (received at least one dose of sorafenib and underwent at least one follow-up assessment) with modification according to toxicity as needed. After administration of sorafenib, patients were followed up in the outpatient clinic after 3 - 4 weeks by an experienced oncologist and hepatologist.

\subsection{Assessment}

Overall survival was the primary outcome of this study. Various clinical parameters including age, sex, aetiology, Child-Pugh class, BCLC stage, presence of portal vein thrombosis-Vascular invasion (VI) and extrahepatic metastasis and adverse effects to sorafenib were analysed. Tumour response was assessed every 8 - 12 weeks by an experienced radiologist who was blind to the patient's information according to the Response Evaluation Criteria in Solid Tumour (RECIST) version 1.0 in 88 patients with available radiological studies. The adverse events records were reviewed using the Common Terminology Criteria for Adverse Events (CTCAE) version 4.0. In this study, patients had the sorafenib discontinued in the presence of radiologic progression, symptomatic progressionor onset of untreatable adverse event.

\subsection{Statistical Analysis}

Data were described by median (25\% quantile-quantile $75 \%$ ) for continuous variables and percentage (frequency) for qualitative variables.

Survival was considered as zero time the start date of use of sorafenib to the event: death or date of last visit. Confirmation of death was obtained through patient records or telephone contact with family members.

Continuous variables were categorized by maximizing the statistical procedure made by log rank and HothornLausen (2003) [18]. The rejection-free survival curves were obtained using the Kaplan-Meier estimator accompanied by the log rank test.

A simple Cox regression was performed to evaluate factors of confusion and interest. The proportionality of hazards was assessed by testing for residues of Schoenfeld (1982) [19].

The variables with $\mathrm{p}$ values $<0.10$ were included in the multivariable Cox regression since they were clinically reasonable and the variables were selected by the backward method.

The association between duration of treatment sorafenib and the clinical and pathological characteristics of the patients was assessed using the Fisher exact test.

$\mathrm{p}$ values $<0.05$ were considered statistically significant variables. All calculations were made using the $\mathrm{R}$ software, version 3.1.2. (R Core Team, 2014) [20].

\section{Results}

There were 64 (73\%) males and 24 (27\%) females included in this study, with a median age of 61.16 years. Eighty (91\%) patients had Child-Pugh class A cirrhosis. Most patients were classified as BCLC C (82\%) in the moment of sorafenib therapy. Hepatitis C virus was the predominant cause of HCC (68\%). Diagnostic methods most common used was CT (66\%). Sorafenib was the initial treatment modality in $43 \%$, followed by TACE in $42 \%$. Baseline characteristics of patients are listed in the Table 1. The most common reason for migration from previous treatments to sorafenib was tumor progression (25\%). The majority of patients were PST 0.

\section{Clinical Parameters Predictive of Overall Survival}

Meantime of sorafenib therapy was 8.23 months (range, 0.4 - 37 months). Sorafenib was permanently discontinued 
Table 1. Baseline characteristics of the patients $(n=88)$.

\begin{tabular}{|c|c|}
\hline Gender & Male \\
\hline$(\%)$ & $64(73 \%)$ \\
\hline \multicolumn{2}{|l|}{ Cirrhosis } \\
\hline$(\%)$ & 84(95\%) \\
\hline \multicolumn{2}{|l|}{ Etiology } \\
\hline $\mathrm{HCV}$ & $60(68 \%)$ \\
\hline Alcohol & $10(11 \%)$ \\
\hline $\mathrm{HBV}$ & $8(9 \%)$ \\
\hline NASH & $4(5 \%)$ \\
\hline Others (hemochromatosis/AIH/fibrolamellar/schistosomiasis) & $6(7 \%)$ \\
\hline \multicolumn{2}{|l|}{ Prior treatment to Sorafenib } \\
\hline TACE & $37(42 \%)$ \\
\hline RFA/PEI & $6(7 \%)$ \\
\hline Resection & $7(8 \%)$ \\
\hline No treatment & $38(43 \%)$ \\
\hline \multicolumn{2}{|l|}{ ChildPugh } \\
\hline A & $80(91 \%)$ \\
\hline B & $8(9 \%)$ \\
\hline \multicolumn{2}{|l|}{ ECOG-Perfomance Status } \\
\hline 0 & $52(59 \%)$ \\
\hline 1 & $30(34 \%)$ \\
\hline 2 & $6(7 \%)$ \\
\hline \multicolumn{2}{|l|}{ Alphafetoprotein } \\
\hline $0-100$ & $41(47 \%)$ \\
\hline$>101$ & 47 (53\%) \\
\hline \multicolumn{2}{|l|}{ BCLC Staging System } \\
\hline B & $16(18 \%)$ \\
\hline $\mathrm{C}$ & $72(82 \%)$ \\
\hline \multicolumn{2}{|l|}{ Number of nodules } \\
\hline 1 & $24(27 \%)$ \\
\hline 2 & $9(10 \%)$ \\
\hline 3 & $12(14 \%)$ \\
\hline Multifocal & 43 (49\%) \\
\hline \multicolumn{2}{|l|}{ Diameter of the nodules } \\
\hline variation & 8 a $190 \mathrm{~mm}$ \\
\hline average & $65.4 \mathrm{~mm}$ \\
\hline median & $64.2 \mathrm{~mm}$ \\
\hline \multicolumn{2}{|l|}{ Metastasis } \\
\hline Yes & 42 (48\%) \\
\hline No & $46(52 \%)$ \\
\hline \multicolumn{2}{|l|}{ Vascular Invasion } \\
\hline Yes & $61(69 \%)$ \\
\hline No & 27 (31\%) \\
\hline
\end{tabular}

HCV—Hepatitis C virus; HBV—Hepatitis B virus; NASH—Non Alcoholic SteatoHepatitis; AIH—Autoimmune Hepatitis; TACE—Transarterial Chemoembolisation; RFA—Radiofrequency Ablation; PEI—Percutaneous Ethanol Injection; BCLC—Barcelona Clinic Liver Cancer staging system.

in 76 patients. The most common cause of discontinuation was disease progression $(n=70)$, followed by deterioration of liver function $(n=15)$. None of the patients discontinued sorafenib because of adverse events.

Table 2 lists the results of univariate and multivariate analysis of potential clinical parameters predictive of 
Table 2. Univariate and multivariate analyses.

\begin{tabular}{|c|c|c|c|c|}
\hline \multirow{2}{*}{ Variable } & \multicolumn{2}{|c|}{ Univariate } & \multicolumn{2}{|c|}{ Multivariate } \\
\hline & HR (CI 95\%) & p value & HR (CI 95\%) & p value \\
\hline Sex & $0.88(0.51-1.54)$ & 0.65 & - & - \\
\hline Age ( $\leq 60$ vs $>60$ years) & $0.65(0.39-1.09)$ & 0.10 & - & - \\
\hline Cirrhosis (yes vs no) & $0.99(0.31-3.19)$ & 0.99 & - & - \\
\hline Prior totreatmentSorafenib & $1.42(0.84-2.38)$ & 0.18 & & \\
\hline Child-Pugh (B vs A) & $1.24(0.56-2.75)$ & 0.59 & & \\
\hline ECOG-PS (2 vs <2) & $2.45(1.01-5.91)$ & 0.04 & & \\
\hline BCLC (C vs B) & $1(0.52-1.93)$ & 0.99 & & \\
\hline Numbers of nodules ( 3 vs $\leq 3$ ) & $0.67(0.36-1.26)$ & 0.21 & & \\
\hline Major nodule ( $\geq 2$ vs $<2 \mathrm{~cm})$ & $0.24(0.08-0.67)$ & $<0.01$ & & \\
\hline Extrahepatic metastasis (yes vs no) & $1.31(0.79-2.17)$ & 0.29 & & \\
\hline Vascular invasion (yes vs no) & $1.23(0.71-2.14)$ & 0.46 & & \\
\hline AFP $(\leq 100$ vs $>100$ ng/mL) & $2.91(1.31-6.48)$ & $<0.01$ & $3.16(1.56-6.41)$ & $<0.01$ \\
\hline Dermatologic adverse event (yes vs no) & $0.35(0.2-0.62)$ & $<0.01$ & $0.44(0.23-0.83)$ & $<0.01$ \\
\hline Diarrhea adverse event (yes vs no) & $0.53(0.32-0.9)$ & $<0.01$ & - & - \\
\hline Time of sorafenib ( $\leq 6 \mathrm{vs}>6$ months) & $0.28(0.16-0.5)$ & $<0.01$ & $0.25(0.14-0.46)$ & $<0.01$ \\
\hline
\end{tabular}

HR—Hazard ratio; CI—Confident interval; $\mathrm{p}<0.05$ vs control.

OS. Age, sex, Child-Pugh class, and presence of prior to sorafenib therapy were factors that did not predict OS. Among the adverse effects, dermatologic and diarrhea were predictive of overall survival in sorafenib-treated HCC patients in univariateanalysis as well as time of use of sorafenib $>6$ months. Mean times of sorafenib therapy were 3.02 months ( $\leq 6$ months, $\mathrm{n}=54$ patients) and 16.5 months ( $>6$ months, $\mathrm{n}=34$ patients). Multivariate analysis of the clinical factors for OS showed that presence of dermatologic adverse effects [HR $=0.4495 \%$ CI $(0.23-0.83) \mathrm{p}<0.01]$ and time of use of sorafenib $>6$ months $[\mathrm{HR}=0.2595 \% \mathrm{CI}(0.14-0.46) \mathrm{p}<0.01]$ were predictive of better OS. While AFP level of $>100 \mathrm{ng} / \mathrm{ml}$ was predictive of worse OS [HR $=3.16$ 95\% CI (1.56 6.41) $\mathrm{p}<0.01]$.

In this study, the median OS was 16.3 months and distributed in OS of $57.31 \%$ and $36.71 \%$ in 1 and 2 years respectively. Dividing them according to the BCLC staging was found: BCLC B: median OS was 10.11 months and distributed in OS of 50\% and 32.8\% in 1 and 2 years, respectively; BCLC C: median OS was 17.22 months and distributed in OS of $59 \%$ and $39.4 \%$ in 1 and 2 years, respectively.

Figure 1 presents predictors of OS according to: AFP level, dermatologic adverse events, duration of sorafenib-treatment (multivariate analysis), diarrhea adverse events and Child Pugh score (univariate analysis).

\section{Discussion}

In the real life settings, we have identified three variables as possible predictors of overall survival in sorafenibtreated advanced HCC patients: Duration of Sorafenib-treatment $>6$ months; presence of dermatologic adverse event and level of alphafetoprotein $<100 \mathrm{ng} / \mathrm{dl}$.

Mean time of Sorafenib-treatment therapy was 8.23 months (range, 0.4 - 37 months) and the median OS was 16.3 months in our study, which is relatively larger than that of previous reports in the literature, like in the SHARP study that were 5.3 months and 10.7 months, respectively [3] [4]. Duration of Sorafenib-treatment $>6$ months have demonstrated better OS ( $\mathrm{p}$ 0.001). We believe that the best management of adverse events may 


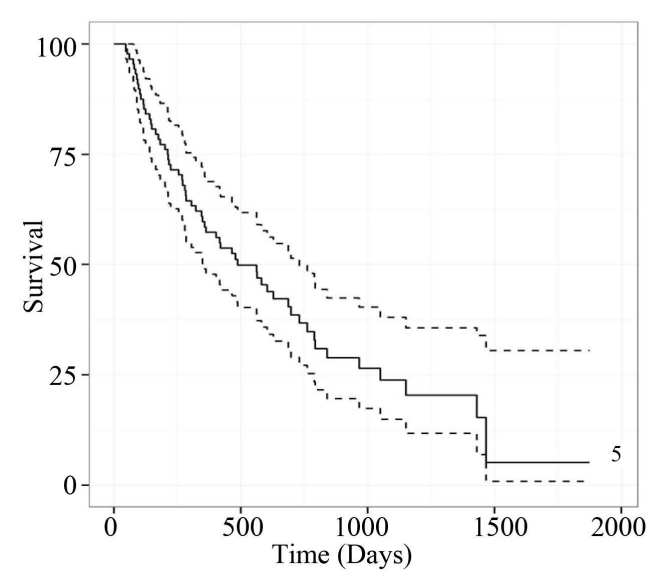

(a)

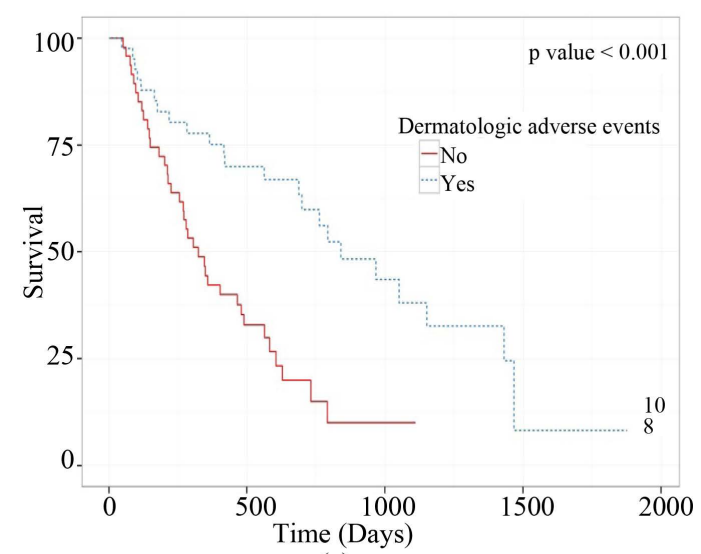

(c)

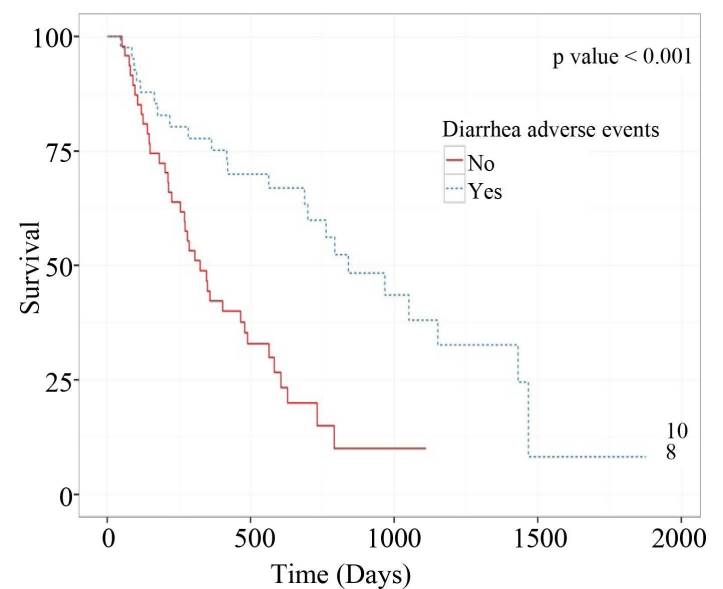

(e)

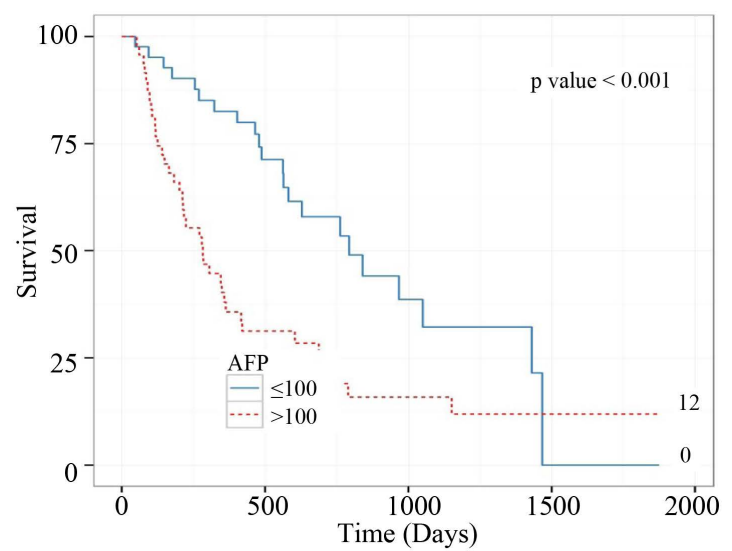

(b)

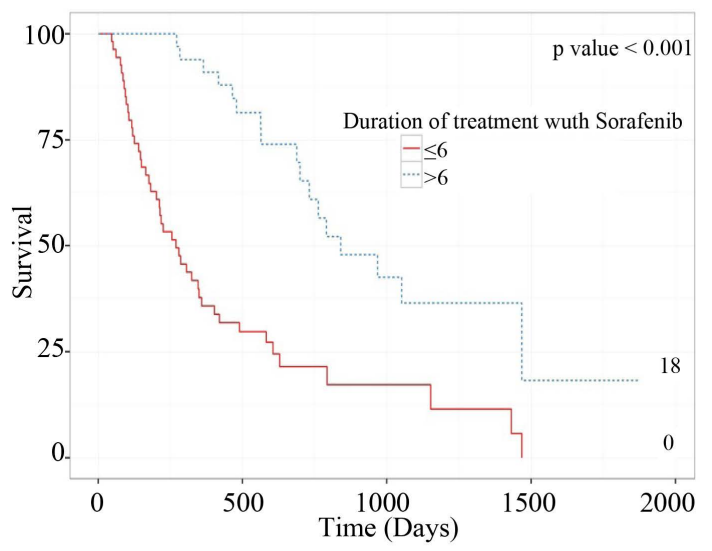

(d)

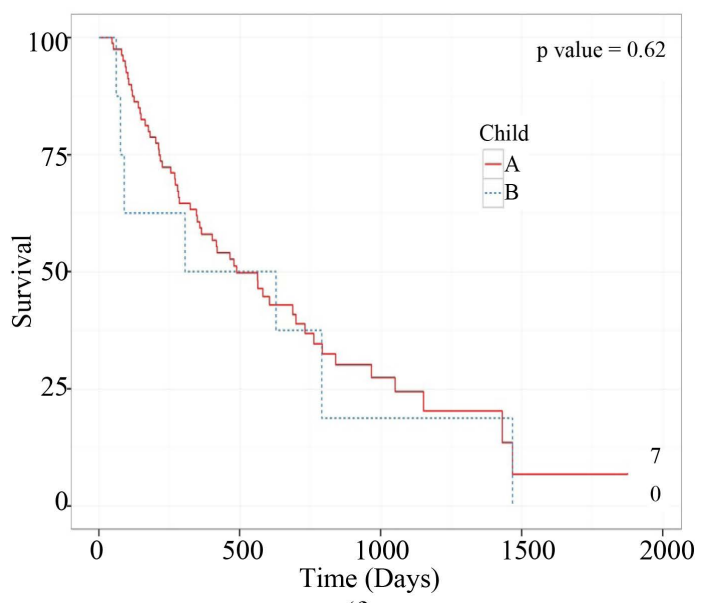

(f)

Figure 1. Predictors of OS in sorafenib-treated advanced HCC patients. (a) Overall survival. (b) Patients with AFP level $<100$ showed better OS. (c) Patients with dermatologic adverse events showed better OS. (d) Patients that used Sorafenib $>6$ months showed better OS. (e) Patients with diarrhea adverse events showed better OS (univariate analysis). (f) There weren’t difference in OS in relation child pugh class.

have contributed to lengthen the time of therapy with sorafenib. Zheng Gang Ren et al. showed that Urea-base cream prophylaxis in patients with advanced HCC starting sorafenib reduced Hand-foot skin reaction (HFSR) rates, extended the time to first occurrence of HFSR, and improved patient quality of life compared with Best Suport Care [21]. The use of urea cream 10\% decrease dermatologic adverse event (DAE) and increase adhe- 
rence to treatment with sorafenib. On one hand "we mask" a clinical parameter of benefit with sorafenib (DAE) but probably increase the effectiveness of treatment for better adhesion. Then, increases the time of treatment with sorafenib and therefore survival.

A point that has been considered in the literature is the time of interruption of sorafenib therapy. The absence of radiological response is not a good indicator of treatment failure and the current recommendation is to maintain treatment until symptomatic progression or onset of untreatable adverse event (AE) [22]. In fact, the reduction of contrast uptake may merely be the result of some vasoconstriction related to the drug itself and not reflect treatment efficacy [23]. When we evaluated patients with the aim to treatment interrupt, many points were observed: Absence of radiological response, variation of alphafetoprotein level, grade of adverse event and symptomatic progression. Perhaps, this care may have contributed to a longer duration of treatment and increased survival.

When trying to identify prognostic factors for longer survival, the presence of dermatologic adverse event (DAE) seemed to be the most impact factors. Prior retrospective studies suggested the presence of DAE as a predictor of OS in HCC patients under sorafenib treatment [10] [24]. In patients who developed skin toxicity in a retrospective study [24] the tumor control rate was $48.3 \%$, versus $19.4 \%$ and the median TTP was 8.1 months versus 4.0 months in the group of patients with and without skin toxicity respectively. This has been confirmed in a prospective study conducted on 147 patients that revealed that the survival of patients with DAEs was significantly better when compared with the rest of the cohort (18.2 vs. 10.1 months, $p=0.009$ ), regardless of baseline characteristics [25]. The results of our study showing a relationship between the development of early DAE during sorafenib therapy and longer OS seem to confirm the role of early cutaneous toxicity as a surrogate marker of efficacy to those observed in that prospective study. In our study, patients with DAE had an increase in the risk of survival of almost three folds of those who did not experience the event.

Diarrhea has been previously described and AE to sorafenib associated with a better treatment response. [26] [27] However, this finding has not been reproduced in more recent study [25] and there is no prospective study confirming this association. In our study, diarrhea was associated with a better overall survival in the univariate analysis. However, the multivariate analysis adjusted by other variables, including DAE, did not confirm the presence of diarrhea as a prognostic factor associated with better survival for patients receiving sorafenib treatment for HCC.

Hollebecque et al. reported an OS of 4.5 months in Child-Pugh class B patients whereas Chiu et al. reported an OS of 2.7 months in Child-Pugh score of 8 and 9 [28] [29]. Kim et al. also reported of worse survival outcome and sorafenib efficacy in Child-Pugh class B patients [30]. In the univariate analysis of the OSbased on Child-Pugh score did not demonstrate a significant difference in our study $(\mathrm{p}=0.62)$. These results might come from relatively small number of patients in the Child class B, and is needed to be investigated in further large scale.

Theoretically, alphafetoprotein (AFP) measurements may be informative for the clinician managing patients at risk for or suffering from hepatocellular carcinoma (HCC) under several different circumstances. These include risk stratification, surveillance, prognosis, and monitoring of response to therapy. However, studies have suggested that for most of these circumstances the value of measuring AFP is limited [31]. Abnormalities in hepatic marker concentrations have been shown to be an adverse prognostic indicator in patients with hepatocellular carcinoma (HCC) [32]. Median overall survival (OS) was shorter in patients with elevated than with normal AFP concentrations, even in the presence of portal vein thrombosis, large or bilobar tumors, or cirrhosis. In a study of 606 patients divided into quartiles by AFP level, median survival was inversely correlated with increasing concentrations of AFP [33]. Furthermore, a recent retrospective analysis of 201 patients with sorafenib-treated, metastatic HCC indicated that serum concentrations of AFP, bilirubin, and albumin were significantly associated with OS and failure-free survival [34]. Personeni et al., suggests that AFP response is an independent surrogate end point for survival that should be considered in conjunction with radiologic response evaluation. In the era of targeted agents, which characteristically increase the rate of disease stabilization, the prognostic role of AFP response warrants further prospective validation in larger series [35].

In this study, AFP level $>100 \mathrm{ng} / \mathrm{ml}$ showed worst prognostic, presenting a greater mortality risk 3.16 (95\% CI 1.56 - 6.41) than an individual with AFP $<10$. While AFP $<100 \mathrm{ng} / \mathrm{ml}$ presents a risk of death 1.44 times higher (95\% CI 0.6 - 3.47). Thus showing, results similar to the literature.

We had some limitations because it is retrospective study, but is a translation of the treatment in real life. We obtained satisfactory results compared to others. 


\section{Conclusion}

In this retrospective study, we had found that advanced HCC patients treated with sorafenib who experienced dermatologic adverse event and AFP level $<100 \mathrm{ng} / \mathrm{ml}$ showed better overall survival. As expected, longer sorafenib therapy ( $\geq 6$ months) was associated to better survival. None of the patients discontinued sorafenib because of adverse events. Even in the presence of adverse events, the use of sorafenib should be continued because the longer usage time improves survival

\section{Conflict of Interest}

The authors who have taken part in this study declared that they do not have anything to disclose regarding funding or conflict of interest with respect to this manuscript. Some of the authors, sometimes, gave private lessons for Bayer.

\section{References}

[1] (2008) International Agency for Cancer Research. GLOBOCAN.

[2] Llovet, J.M., Bru, C. and Bruix, J. (1999) Prognosis of Hepatocellular Carcinoma: The BCLC Staging Classification. Seminars in Liver Disease, 19, 329-338. http://dx.doi.org/10.1055/s-2007-1007122

[3] Llovet, J.M., Ricci, S., Mazzaferro, V., Hilgard, P., Gane, E., Blanc, J.F., de Oliveira, A.C., Santoro, A., Raoul, J.L., Forner, A., Schwartz, M., Porta, C., Zeuzem, S., Bolondi, L., Greten, T.F., Galle, P.R., Seitz, J.F., Borbath, I., Haussinger, D., Giannaris, T., Shan, M., Moscovici, M., Voliotis, D. and Bruix, J. (2008) Sorafenib in Advanced Hepatocellular Carcinoma. New England Journal of Medicine, 359, 378-390. http://dx.doi.org/10.1056/NEJMoa0708857

[4] Cheng, A.L., Kang, Y.K., Chen, Z., Tsao, C.J., Qin, S., Kim, J.S., Luo, R., Feng, J., Ye, S., Yang, T.S., Xu, J., Sun, Y., Liang, H., Liu, J., Wang, J., Tak, W.Y., Pan, H., Burock, K., Zou, J., Voliotis, D. and Guan, Z. (2009) Efficacy and Safety of Sorafenib in Patients in the Asia-Pacific Region with Advanced Hepatocellular Carcinoma: A Phase III Randomised, Double-Blind, Placebo-Controlled Trial. The Lancet Oncology, 10, 25-34. http://dx.doi.org/10.1016/S1470-2045(08)70285-7

[5] Bruix, J., Raoul, J.L., Sherman, M., Mazzaferro, V., Bolondi, L., Craxi, A., et al. (2012) Efficacy and Safety of Sorafenib in Patients with Advanced Hepatocellular Carcinoma: Subanalyses of a Phase III Trial. Journal of Hepatology, 57, 821-829. http://dx.doi.org/10.1016/j.jhep.2012.06.014

[6] Scandurra, G., Aiello, R.A., Ali, M., et al. (2012) Appropriate Management of Cutaneous Adverse Events Maximizes Compliance with Sorafenib Treatment: A Single-Centre Experience. Future Oncology, 8, 609-615. http://dx.doi.org/10.2217/fon.12.35

[7] Llovet, J.M., Pena, C.E., Lathia, C.D., Shan, M., Meinhardt, G. and Bruix, J. (2012) Plasma Biomarkers as Predictors of Outcome in Patients with Advanced Hepatocellular Carcinoma. Clinical Cancer Research, 18, 2290-2300. http://dx.doi.org/10.1158/1078-0432.CCR-11-2175

[8] Zhang, Z., Zhou, X., Shen, H., Wang, D. and Wang, Y. (2009) Phosphorylated ERK Is a Potential Predictor of Sensitivity to Sorafenib When Treating Hepatocellular Carcinoma: Evidence from an in Vitro Study. BMC Medicine, 7, 41. http://dx.doi.org/10.1186/1741-7015-7-41

[9] Pinter, M., Sieghart, W., Hucke, F., et al. (2011) Prognostic Factors in Patients with Advanced Hepatocellular Carcinoma Treated with Sorafenib. Alimentary Pharmacology \& Therapeutics, 34, 949-959. http://dx.doi.org/10.1111/j.1365-2036.2011.04823.x

[10] Otsuka, T., Eguchi, Y., Kawazoe, S., et al. (2012) Skin Toxicities and Survival in Advanced Hepatocellular Carcinoma Patients Treated with Sorafenib. Hepatology Research, 42, 879-886. http://dx.doi.org/10.1111/j.1872-034X.2012.00991.X

[11] Shao, Y.Y., Ho, M.C., Cheng, A.L. and Hsu, C.H. (2014) Long-Term Disease-Free Survival Achieved by Anti-Angiogenic Therapy plus Surgery in a Hepatocellular Carcinoma Patient with Extensive Liver Involvement and Lung Metastases. Journal of the Formosan Medical Association, 113, 577-578.

[12] Du, J., Qian, X. and Liu, B. (2013) Long-Term Progression-Free Survival in a Case of Hepatocellular Carcinoma with Vertebral Metastasis Treated with a Reduced Dose of Sorafenib: Case Report and Review of the Literature. Oncology Letters, 5, 381-385.

[13] Curtit, E., Thiery-Vuillemin, A., Nguyen, T., Heyd, B., Pivot, X., Martino, V.D. and Borg, C. (2011) Complete Histologic Response Induced by Sorafenib in Advanced Hepatocellular Carcinoma: A Case Report. Journal of Clinical Oncology, 29, e330-e332. http://dx.doi.org/10.1200/JCO.2010.32.6785

[14] Bruix, J. and Sherman, M. (2011) Management of Hepatocellular Carcinoma: An Update. Hepatology, 53, $1020-1022$. 
http://dx.doi.org/10.1002/hep.24199

[15] Dufour, J.F., Greten, T.F., Raymond, E., Roskams, T., De, T., Ducreux, M., et al. (2012) EASL-EORTC Clinical Practice Guidelines: Management of Hepatocellular Carcinoma. Journal of Hepatology, 56, 908-943. http://dx.doi.org/10.1016/j.jhep.2011.12.001

[16] Oken, M.M., Creech, R.H., Tormey, D.C., Horton, J., Davis, T.E., McFadden, E.T., et al. (1982) Toxicity and Response Criteria of the Eastern Cooperative Oncology Group. American Journal of Clinical Oncology, 5, 649-655. http://dx.doi.org/10.1097/00000421-198212000-00014

[17] Pugh, R.N.H., Murray-Lyon, I.M., Dawson, J.L., Pietroni, M.C. and Williams, R. (1973) Transection of the Oesophagus for Bleeding Oesophageal Varices. British Journal of Surgery, 60, 646-649. http://dx.doi.org/10.1002/bjs.1800600817

[18] Hothorn, T. and Lausen, B. (2003) On the Exact Distribution of Maximally Selected Rank Statistics. Computational Statistics \& Data Analysis, 43, 121-137. http://dx.doi.org/10.1016/S0167-9473(02)00225-6

[19] Schoenfeld, D. (1982) Partial Residuals for the Proportional Hazards Regression Model. Biometrika, 69, $239-241$. http://dx.doi.org/10.1093/biomet/69.1.239

[20] R Core Team (2014) R: A Language and Environment for Statistical Computing. R Foundation for Statistical Computing, Vienna. http://www.R-project.org/

[21] Ren, Z., Zhu, K., Kang, H., Lu, M., Qu, Z., Lu, L., Song, T., Zhou, W., Wang, H., Yang, W., Wang, X., Yang, Y., Shi, L., Bai, Y., Guo, X. and Ye, S.L. (2015) Randomized Controlled Trial of the Prophylactic Effect of Urea-Based Cream on Sorafenib-Associated Hand-Foot Skin Reactions in Patients with Advanced Hepatocellular Carcinoma. Journal of Clinical Oncology, 33, 894-900. http://dx.doi.org/10.1200/JCO.2013.52.9651

[22] Reig, M., Darnell, A., Forner, A., Rimola, J., Ayuso, C. and Bruix, J. (2014) Systemic Therapy for Hepatocellular Carcinoma: The Issue of Treatment Stage Migration and Registration of Progression Using the BCLC-Refined RECIST. Seminars in Liver Disease, 34, 444-455. http://dx.doi.org/10.1055/s-0034-1394143

[23] Reig, M., Rimola, J., Torres, F., Darnell, A., Rodriguez-Lope, C., Forner, A., et al. (2013) Postprogression Survival of Patients with Advanced Hepatocellular Carcinoma: Rationale for Second-Line Trial Design. Hepatology, 58, 20232031. http://dx.doi.org/10.1002/hep.26586

[24] Vincenzi, B., Santini, D., Russo, A., Addeo, R., Giuliani, F., Montella, L., et al. (2010) Early Skin Toxicity as a Predictive Factor for Tumor Control in Hepatocellular Carcinoma Patients Treated with Sorafenib. Oncologist, 15, 85-92. http://dx.doi.org/10.1634/theoncologist.2009-0143

[25] Reig, M., Torres, F., Rodriguez-Lope, C., Forner, A., LLarch, N., Rimola, J., et al. (2014) Early Dermatologic Adverse Events Predict Better Outcome in HCC Patients Treated with Sorafenib. Journal of Hepatology, 61, 318-324. http://dx.doi.org/10.1016/j.jhep.2014.03.030

[26] Yada, M., Masumoto, A., Motomura, K., Tajiri, H., Morita, Y., Suzuki, H., et al. (2014) Indicators of Sorafenib Efficacy in Patients with Advanced Hepatocellular Carcinoma. World Journal of Gastroenterology, 20, 12581-12587. http://dx.doi.org/10.3748/wjg.v20.i35.12581

[27] Cho, J.Y., Paik, Y.H., Lim, H.Y., Kim, Y.G., Lim, H.K., Min, Y.W., et al. (2013) Clinical Parameters Predictive of Outcomes in Sorafenib-Treated Patients with Advanced Hepatocellular Carcinoma. Liver International, 33, 950-957. http://dx.doi.org/10.1111/liv.12168

[28] Hollebecque, A., Cattan, S., Romano, O., et al. (2011) Safety and Efficacy of Sorafenib in Hepatocellular Carcinoma: The Impact of the Child-Pugh Score. Alimentary Pharmacology \& Therapeutics, 34, 1193-1201. http://dx.doi.org/10.1111/j.1365-2036.2011.04860.x

[29] Chiu, J., Tang, Y.F., Yao, T.J., et al. (2012) The Use of Single-Agent Sorafenib in the Treatment of Advanced Hepatocellular Carcinoma Patients with Underlying Child-Pugh B Liver Cirrhosis: A Retrospective Analysis of Efficacy, Safety and Survival Benefits. Cancer, 118, 5293-5301. http://dx.doi.org/10.1002/cncr.27543

[30] Kim, J.E., Ryoo, B.Y., Ryu, M.H., et al. (2011) Sorafenib for Hepatocellular Carcinoma According to Child-Pugh Class of Liver Function. Cancer Chemotherapy and Pharmacology, 68, 1285-1290. http://dx.doi.org/10.1007/s00280-011-1616-x

[31] Sherman, M. (2010) The Resurrection of Alpha-Fetoprotein. Journal of Hepatology, 52, 939-940.

[32] Carr, B.I., Pancoska, P. and Branch, R.A. (2009) Tumor and Liver Determinants of Prognosis in Unresectable Hepatocellular Carcinoma: A Large Case Cohort Study. Hepatology International, 4, 396-405. http://dx.doi.org/10.1007/s12072-009-9157-7

[33] Nomura, F., Ohnishi, K. and Tanabe, Y. (1989) Clinical Features and Prognosis of Hepatocellular Carcinoma with Reference to Serum Alpha-Fetoprotein Levels: Analysis of 606 Patients. Cancer, 64, 1700-1707. http://dx.doi.org/10.1002/1097-0142(19891015)64:8<1700::AID-CNCR2820640824>3.0.CO;2-Z 
[34] Baek, K.K., Kim, J.H., Uhm, J.E., Park, S.H., Lee, J., Park, J.O., et al. (2011) Prognostic Factors in Patients with Advanced Hepatocellular Carcinoma Treated with Sorafenib: A Retrospective Comparison with Previously Known Prognostic Models. Oncology, 80, 167-174. http://dx.doi.org/10.1159/000327591

[35] Personeni, N., Bozzarelli, S., Pressiani, T., Rimassa, L., Tronconi, M.C., Sclafani, F., Carnaghi, C., Pedicini, V., Giordano, L. and Santoro, A. (2012) Usefulness of Alpha-Fetoprotein Response in Patients Treated with Sorafenib for Advanced Hepatocellular Carcinoma. Journal of Hepatology, 57, 101-107. 